\title{
Quantum Stark broadening of Ar XV lines. Strong collision and quadrupolar potential contributions
}

\author{
H. Elabidi ${ }^{a, b, *}$, S. Sahal-Bréchot ${ }^{\mathrm{c}}$, M. S. Dimitrijevićc, ${ }^{\mathrm{c}, \mathrm{d}}$ \\ ${ }^{a}$ Deanship of the Foundation Year, Umm Al-Qura University, Makkah, KSA \\ ${ }^{b}$ GRePAA, Faculté des Sciences de Bizerte, Université de Carthage, Tunisia \\ ${ }^{c}$ LERMA, Obs. Paris, UMR CNRS 8112, UPMC, Bâtiment Evry Schatzman, France \\ ${ }^{d}$ Astronomical Observatory, Volgina 7, 11060 Belgrade 38, Serbia
}

\begin{abstract}
We present in this paper electron impact broadening for six Ar XV lines using our quantum mechanical formalism and the semiclassical perturbation one. Additionally, our calculations of the corresponding atomic structure data (energy levels and oscillator strengths) and collision strengths are given as well. The lines considered here are divided into two 'sets: a first set of four lines involving the ground level: $1 \mathrm{~s}^{2} 2 \mathrm{~s}^{2}{ }^{1} \mathrm{~S}_{0}-1 \mathrm{~s}^{2} 2 \mathrm{~s} n \mathrm{p}{ }^{1} \mathrm{P}_{1}^{\mathrm{o}}$ where $2 \leq n \leq 5$ and a second set of two lines involving excited levels: $1 \mathrm{~s}^{2} 2 \mathrm{~s} 2 \mathrm{p}{ }^{1} \mathrm{P}_{1}^{\mathrm{o}}-1 \mathrm{~s}^{2} 2 \mathrm{~s} 3 \mathrm{~s}{ }^{1} \mathrm{~S}_{0}$ and $1 \mathrm{~s}^{2} 2 \mathrm{~s} 2 \mathrm{p}{ }^{3} \mathrm{P}_{0}^{\mathrm{o}}-1 \mathrm{~s}^{2} 2 \mathrm{~s} 3 \mathrm{~s}{ }^{3} \mathrm{~S}_{1}$. An extensive comparison between the quantum and the semiclassical results was performed in order to analyze the reason for differences between quantum and semiclassical results up to the factor of two. It has been shown that the difference between the two results may be due to the evaluation of strong collision contributions by the semiclassical formalism. Except few semiclassical results, the present results are the first to be published. After the recent discovery of the far UV lines of Ar VII in the spectra of very hot central stars of planetary nebulae and white dwarfs, the present -and may be further- results can be used also for the corresponding future spectral analysis.
\end{abstract}

'Keywords:

line profiles; stars atmospheres; white dwarfs; quantum formalism

\section{Introduction}

The Stark broadening mechanism is important in stellar spectroscopy and in the analysis of astrophysical and laboratory plasmas. Its influence should be considered for the opacity calculations, the modelling of stellar interiors, the estimation of radiative transfer through the stellar plasmas and for the determination of chemical abundances of elements (Dimitrijević, 2003). The need for spectral line broadening calculations is stimulated by the development of computers. Moreover, the development of instruments 'and space astronomy, such as the new $\mathrm{X}$-ray space telescope Chandra, stimulated the calculations of line broad'ening of trace elements in the $\mathrm{X}$-ray wavelength range. Barstow et al. (1998) have shown that analysis of white 'dwarf atmospheres, where Stark broadening is dominant compared to the thermal Doppler broadening, needs models taking into account heavy element opacity. Consequently, atomic and line broadening data for many elements are needed for stellar plasma research. The recent discovery of the far UV lines of Ar VII in the spectra

\footnotetext{
* Corresponding author

Email addresses: haelabidi@uqu.edu.sa, haykel.elabidi@fsb.rnu.tn (H. Elabidi), sylvie.sahal-brechot@obspm.fr (S. Sahal-Bréchot), mdimitrijevic@aob.rs (M. S. Dimitrijević)
}

of very hot central stars of planetary nebulae and white dwarfs (Werner et al., 2007) showed the astrophysical interest for atomic and line broadening data for this element in various ionization stages. Ar XV is one of these important ions. The only Ar XV line broadening calculations existing in the literature are the semiclassical ones (Dimitrijević et al., 2012), where the authors claimed that there are no experimental or other theoretical results for a comparison.

The calculations performed in the present paper are based on the quantum mechanical approach and the semiclassical perturbation one. The quantum mechanical expression for electron impact broadening calculations for intermediate coupling was obtained in Elabidi et al. (2004). We performed the first calculations for the $2 s 3 s-2 s 3 p$ transitions in Be-like ions from nitrogen to neon (Elabidi et al., 2007, 2008a) and for the $3 s-3 p$ transitions in Li-like ions from carbon to phosphor (Elabidi et al., 2008b, 2009). This approach was also used in Elabidi and Sahal-Bréchot (2011) to check the dependence on the upper level ionization potential of electron impact widths, and in Elabidi et al. (2011) to provide some missing line broadening data for the C IV, N VI, O VI and F VII resonance lines. In our quantum approach, all the parameters required for the calculations of the line broadening such as radiative atomic data (energy levels, oscillator strengths...) 
or collisional data (collision strengths or cross sections, scattering matrices...) are evaluated during the calculation and not taken from other data sources. We used the sequence of the UCL atomic codes SUPERSTRUCTURE/DW/JAJOM that have been used for many years to provide fine structure wavefunctions, energy levels, wavelengths, radiative probability rates and electron impact collision strengths. Recently they have been adapted to perform line broadening calculations (Elabidi et al., 2008a). The semiclassical perturbation formalism is described in Sahal-Bréchot (1969a,b, 1974); Fleurier et al. (1977) and updated by Dimitrijević and Sahal-Bréchot (1984, 1995). The atomic structure data (energy levels and oscillator strengths) used by the semiclassical formalism for the evaluation of line broadening are taken from the code SUPERSTRUCTURE (Eissner et al., 1974).

We will analyze here as well the reasons for discrepancies (up to factor 2) between results for electron broadening of isolated non hydrogenic ion lines obtained with semiclassical and quantum methods as as were used by Ralchenko et al. (2001, 2003); Alexiou and Lee (2006). For example Ralchenko et al. (2001) obtained, using quantummechanical method, electron-impact widths of the $2 \mathrm{~s} 3 \mathrm{~s}-2 \mathrm{~s} 3 \mathrm{p}$ singlet and triplet lines of the beryllium-like ions from $\mathrm{B}$ II to $\mathrm{O} \mathrm{V}$, and found that their results are generally smaller from most semiclassical widths. In Ralchenko et al. (2003), the similar conclusion was obtained for electronimpact widths of the $3 \mathrm{~s}-3 \mathrm{p}$ transitions in Li-like ions from B III to Ne VIII. It was also found that the difference between experimental and quantum results monotonically increases with the spectroscopic charge of an ion. Alexiou and Lee (2006) investigated the reasons for discrepancies of electron-impact widths of isolated ion lines, obtained with semiclassical non-perturbative and fully quantum closecoupling and convergent close-coupling calculations, and they concluded that the major reason is the neglect of penetration by the semiclassical calculations. They also obtained and analyzed data for Li-like $3 \mathrm{~s}-3 \mathrm{p}$ from Be III to Ne VIII, Be-like $2 \mathrm{~s} 3 \mathrm{~s}{ }^{3} \mathrm{~S}-2 \mathrm{~s} 3 \mathrm{p}^{3} \mathrm{P}$ from C III to Ne VII and Be-like $2 \mathrm{~s} 3 \mathrm{~s}{ }^{1} \mathrm{~S}-2 \mathrm{~s} 3 \mathrm{p}^{1} \mathrm{P}$ from $\mathrm{N}$ IV to $\mathrm{N}$ VII. In order to contribute to the clarification of this problem, it is of interest to compare quantum and semiclassical results and for a more highly charged ion like $\mathrm{Ar} \mathrm{XV}$, which is one of objectives of the present work.

In the present paper, Stark widths for six Ar XV lines will be calculated using the two described formalisms and an extensive comparison between the two results will be performed, in order to contribute to the explanation of reasons for discrepancies found in some cases, for line widths for ions in lower ionization stages than Ar XV. It is also of interest to compare two methods for such a higher ionization stage. Besides the Stark broadening data, we will present the results of our calculations of the corresponding atomic structure data (energy levels and oscillator strengths) and collision strengths.

\section{Outline of the quantum approach and compu- tational procedure}

We present here an outline of the quantum formalism of electron impact broadening. More details have been given elsewhere (Elabidi et al., 2004, 2008a). The calculations are made within the framework of the impact approximation, which means that the time interval between collisions is much longer than the duration of a collision. The expression of the Full Width at Half Maximum $W$ obtained in Elabidi et al. (2008a) is :

$$
\begin{aligned}
W= & 2 N_{e}\left(\frac{\hbar}{m}\right)^{2}\left(\frac{2 m \pi}{k_{B} T}\right)^{\frac{1}{2}} \\
& \times \int_{0}^{\infty} \Gamma_{w}(\varepsilon) \exp \left(-\frac{\varepsilon}{k_{B} T}\right) d\left(\frac{\varepsilon}{k_{B} T}\right),
\end{aligned}
$$

where $k_{B}$ is the Boltzmann constant, $N_{e}$ is the electron density, $T$ is the electron temperature and

$$
\begin{aligned}
\Gamma_{w}(\varepsilon)= & \sum_{J_{i}^{T} J_{f}^{T} l K_{i} K_{f}} \frac{\left[K_{i}, K_{f}, J_{i}^{T}, J_{f}^{T}\right]}{2} \\
& \times\left\{\begin{array}{c}
J_{i} K_{i} l \\
K_{f} J_{f} 1
\end{array}\right\}^{2}\left\{\begin{array}{c}
K_{i} J_{i}^{T} s \\
J_{f}^{T} K_{f} 1
\end{array}\right\}^{2} \\
& \times\left[1-\left(\operatorname{Re}\left(S_{i}\right) \operatorname{Re}\left(S_{f}\right)+\operatorname{Im}\left(S_{i}\right) \operatorname{Im}\left(S_{f}\right)\right)\right],
\end{aligned}
$$

where $L_{i}+S_{i}=J_{i}, J_{i}+l=K_{i}$ and $K_{i}+s=J_{i}^{T} . L$ and $S$ represent the atomic orbital angular momentum and spin of the target, $l$ is the electron orbital momentum, the superscript $T$ denotes the quantum numbers of the total electron+ion system. $S_{i}\left(S_{f}\right)$ are the scattering matrix elements for the initial (final) levels, expressed in the intermediate coupling approximation, $\operatorname{Re}(\mathrm{S})$ and $\operatorname{Im}(\mathrm{S})$ are respectively the real and the imaginary parts of the $\mathrm{S}$ matrix element, $\left\{\begin{array}{l}a b c \\ d e f\end{array}\right\}$ represent $6-\mathrm{j}$ symbols and we adopt the notation $[x, y, \ldots]=(2 x+1)(2 y+1) \ldots$ Both $S_{i}$ and $S_{f}$ are calculated for the same incident electron energy $\varepsilon=m v^{2} / 2$. The equation (1) takes into account fine structure effects and relativistic corrections resulting from the breakdown of the $L S$ coupling approximation for the target.

The atomic structure and the collisional data are needed for line broadening evaluation. The atomic structure in intermediate coupling is performed through the SUPERSTRUCTURE code (SST) (Eissner et al., 1974). The scattering problem in $L S$ coupling is carried out by the DISTORTED WAVE (DW) code (Eissner, 1998) as in Elabidi et al. (2008a). This weak coupling approximation for the collision part assumed in DW is adequate for highly charged ions colliding with electrons since the close collisions are of small importance. The JAJOM code (Saraph, 1978) is used for the scattering problem in intermediate coupling. $\mathbf{R}$-matrices in intermediate coupling and real 
$(\operatorname{Re} \mathbf{S})$ and imaginary part $(\operatorname{Im} \mathbf{S})$ of the scattering matrix $\mathbf{S}$ have been calculated using the transformed version of JAJOM (Elabidi \& Dubau, unpublished results) and the program RtoS (Dubau, unpublished results) respectively. The evaluation of $\operatorname{Re} \mathbf{S}$ and $\operatorname{Im} \mathbf{S}$ is done according to:

$$
\operatorname{Re} \mathbf{S}=\left(1-\mathbf{R}^{2}\right)\left(1+\mathbf{R}^{2}\right)^{-1}, \operatorname{Im} \mathbf{S}=2 \mathbf{R}\left(1+\mathbf{R}^{2}\right)^{-1}
$$

The relation $\mathbf{S}=(\mathbf{1}+\mathbf{i R})(\mathbf{1}-\mathbf{i R})^{-\mathbf{1}}$ guarantees the unitarity of the $\mathbf{S}$-matrix.

\section{Results and discussion}

We present in the following subsections some atomic data and line broadening data for Ar XV. Energy levels are compared to the available theoretical (Bhatia and Landi, 2008; Kramida et al., 2012) and experimental (Edlen, 1983, 1985; Khardi et al., 1994; Lepson et al., 2003) results. Oscillator strengths and collision strengths for the $\mathrm{Ar} \mathrm{XV}$ lines are compared to the available theoretical results (Bhatia and Landi, 2008). Electron impact full widths at half maximum (FWHM) in $\AA(W=2 w)$ for the considered $\mathrm{Ar}$ $\mathrm{XV}$ lines are calculated for a range of electron temperatures from $5 \times 10^{5} \mathrm{~K}$ to $2 \times 10^{6} \mathrm{~K}$ and for an electron density of $10^{20} \mathrm{~cm}^{-3}$. We choose four lines involving the ground level $\left(1 \mathrm{~s}^{2} 2 \mathrm{~s}^{2}{ }^{1} \mathrm{~S}_{0}-1 \mathrm{~s}^{2} 2 \mathrm{~s} n \mathrm{p}{ }^{1} \mathrm{P}_{1}^{\mathrm{o}}\right.$ where $\left.2 \leq n \leq 5\right)$ and two others involving excited levels $\left(1 \mathrm{~s}^{2} 2 \mathrm{~s} 2 \mathrm{p}{ }^{1} \mathrm{P}_{1}^{\mathrm{o}}-1 \mathrm{~s}^{2} 2 \mathrm{~s} 3 \mathrm{~s}{ }^{1} \mathrm{~S}_{0}\right.$ and $\left.1 \mathrm{~s}^{2} 2 \mathrm{~s} 2 \mathrm{p}{ }^{3} \mathrm{P}_{0}^{\mathrm{o}}-1 \mathrm{~s}^{2} 2 \mathrm{~s} 3 \mathrm{~s}{ }^{3} \mathrm{~S}_{1}\right)$. Calculations are based on the quantum mechanical and the semiclassical perturbation formalisms.

\subsection{Structure and electron scattering data}

The configurations used in the atomic structure description are $1 \mathrm{~s}^{2}\left(2 \mathrm{~s}^{2}, 2 \mathrm{~s} 2 \mathrm{p}, 2 \mathrm{p}^{2}, 2 \mathrm{~s} n l\right)$ where $3 \leq n \leq 5$ and $l=\mathrm{s}, \mathrm{p}, \mathrm{d}$. This set of configurations gives rise to 118 fine structure levels. In the code SST, the wave functions are determined by diagonalization of the non relativistic Hamiltonian using orbitals calculated in a scaled ThomasFermi-Dirac Amaldi (TFDA) potential. The scaling parameters for this potential $\left(\lambda_{l}\right)$ have been obtained by a self-consistent energy minimization procedure, in our case on all term energies of the 21 configurations. Relativistic corrections (spin-orbit, mass, Darwin and one-body) are also introduced in SST.

We perform a comparison of our energy levels and oscillator strengths to those published by Bhatia and Landi (2008) and in the database NIST (Kramida et al., 2012). This preliminary comparison is important since the accuracy of the atomic structure (especially the oscillator strengths) is a prerequisite for the accuracy of the line broadening results. We present in Table 1, energy levels for the lowest 20 levels belonging to the configurations $1 \mathrm{~s}^{2}\left(2 \mathrm{~s}^{2}, 2 \mathrm{~s} 2 \mathrm{p}, 2 \mathrm{p}^{2}, 2 \mathrm{~s} 3 \mathrm{~s}, 2 \mathrm{~s} 3 \mathrm{p}, 2 \mathrm{~s} 3 \mathrm{~d}\right)$. Our energies are compared to the experimental ones (Edlen, 1983, 1985; Khardi et al., 1994; Lepson et al., 2003), to the 27-configuration model of Bhatia and Landi (2008) and to the NIST (Kramida et al., 2012) values, and an excellent agreement (the difference is less than $1 \%$ ) has been found between the three results showing that our 21-configuration model provides acceptable atomic structure data. Oscillator strengths for some transitions from the first five levels to the lowest ten levels (belonging to the configurations $2 \mathrm{~s}^{2}, 2 \mathrm{~s} 2 \mathrm{p}$ and $2 \mathrm{p}^{2}$ ) are presented in Table 2 and compared to the 27configuration model of Bhatia and Landi (2008). The relative difference between the two results is about $10 \%$. We can conclude from the preceding comparisons that our atomic structure study is sufficiently accurate to be adopted in the scattering problem and thus in the line broadening calculations. Collision strengths for the same transitions as for the oscillator strengths are presented in Table 2. Comparison has been made with the 27-configuration results of Bhatia and Landi (2008) and an overall reasonable agreement has been found between the two results. In some cases, notable differences appear especially for the energy $180 \mathrm{Ry}$. We can note the case of the transition $1-5\left(2 \mathrm{~s}^{2}{ }^{1} \mathrm{~S}_{0}-2 \mathrm{~s} 2 \mathrm{p}{ }^{1} \mathrm{P}_{0}^{\mathrm{o}}\right)$ which is an optical allowed transition, and it is shown in Elabidi et al. (2012) that for such transitions, whose energy difference $\Delta E$ is very small, collision strengths can not converge at low total angular momentum $J^{T}$ especially at high electron energies.

In our line broadening calculations (Eq. 1), we use the imaginary and the real parts of the scattering matrices and these parameters are related to the corresponding collision strengths. Consequently, the accuracy of collision strengths presented in Table 2 is very important for the accuracy of our line broadening data.

\subsection{Line broadening data}

We present in Table 3 widths of the four lines: $2 \mathrm{~s}^{2}$ ${ }^{1} \mathrm{~S}_{0}-2 \operatorname{sip}{ }^{1} \mathrm{P}_{1}^{\mathrm{o}}$ where $2 \leq n \leq 5$ involving the ground level, and in Table 3 the two lines $2 \mathrm{~s} 2 \mathrm{p}{ }^{1} \mathrm{P}_{1}^{\mathrm{o}}-2 \mathrm{~s} 3 \mathrm{~s}{ }^{1} \mathrm{~S}_{0}$ and $2 \mathrm{~s} 2 \mathrm{p}{ }^{3} \mathrm{P}_{0}^{\mathrm{o}}-2 \mathrm{~s} 3 \mathrm{~s}{ }^{3} \mathrm{~S}_{1}$ involving excited levels. Calculations are based on the quantum mechanical (Q) and the semiclassical perturbation (SCP) formalisms. We note that for all these transitions, the SCP results are overestimated compared to the quantum ones, and the average relative difference is about $70 \%$. We note also that, except for the resonance line, the two results $\mathrm{Q}$ and SCP become close to each other with the increase of the principal quantum number $n$. Table 4 shows that for transitions that do not involve the ground level, the SCP results are no longer higher than the quantum ones. We found also that the disagreement between the two results is less for these transitions: the quantum results are about $33 \%$ higher than the SCP ones.

To explain at least a part of the previous behaviour of line widths with the principal quantum number $n$, we present in Table 5 the contributions of strong collisions and those of the quadrupolar potential for all the considered transitions. We note that, in general (except for the resonance line), the contributions of strong collisions and those of the quadrupolar potential are important for transitions 
involving levels with low principal quantum number $n$. Inelastic collisions due to more distant collisions are quite negligible. We found also that when the contributions of strong and close collisions, and thus the contributions of the elastic collisions due to the quadrupolar potential is dominant, the disagreement between the SCP and the quantum results is important. For example, for transitions between excited levels (the two last ones in Table 5), the relative difference between the $\mathrm{SCP}$ and the quantum results are about $25 \%$. In these cases, the strong collisions and the quadrupolar potential have the lowest contributions (respectively $35 \%$ and $56 \%$ ) compared to the four other transitions. This behaviour can be explained by the use of the hydrogenic model for the atomic structure in the SCP formalism to evaluate the quadrupolar potential. It is known that this approach overestimates the corresponding contributions to line widths.

\section{Conclusion}

We have calculated in this work, atomic structure data (energy levels and oscillator strengths), collision strengths and electron impact broadening for Ar XV. To check their accuracy, comparisons of our level energies with the experimental (Edlen, 1983, 1985; Khardi et al., 1994; Lepson et al., 2003) and with the theoretical (Bhatia and Landi, 2008; Kramida et al., 2012) results have been performed and a relative difference of about $1 \%$ has been found. Our oscillator strengths have been compared to those of Bhatia and Landi (2008), and we found that the two results agree within $10 \%$. For collision strengths, an overall agreement has been found between our results and those of Bhatia and Landi (2008). This shows firstly that we can trust our preliminary data and that they can be used with confidence in our line broadening calculations. For line broadening, several important results can be derived from our study. Firstly, we find that the disagreement between the semiclassical and the quantum results is important when the contributions of strong and close collisions, and thus the contributions of the elastic collisions due to the quadrupolar potential are dominant. In these cases, the semiclassical results are always higher than the quantum ones. Secondly, we remark that the contributions of such elastic collisions are important for transitions involving levels with low principal quantum numbers $n$ (except for the resonance line). Another point is that for transitions that do not involve the ground level (for which the contributions of strong collisions are the smallest), the SCP results are no longer higher than the quantum ones. Finally, we can explain the overestimation of the semiclassical line widths compared to the quantum ones by the fact that the semiclassical formalism uses the hydrogenic approximation to evaluate the quadrupolar potential. Extensive works on the strong collision contributions to the line widths and their behaviour with the ionization stages along some isoelectronic sequences will be welcome to in- vestigate their effects on lines broadening and to study their evaluation in the semiclassical formalism.

\section{Acknowledgments}

This work has been supported by the Tunisian research unit 05/UR/12-04. It is also a part of the project 176002 "Influence of collisional processes on astrophysical plasma line shapes" supported by the Ministry of Education, Science and Technological Development of Serbia.

\section{References}

Alexiou, S. \& Lee, R.W., Semiclassical calculations of line broadening in plasmas: Comparison with quantal results, J. Quant. Spectrosc. Radiat. Tranfer, 99, 10-20, 2006.

Barstow M.A., Hubeny I. \& Holberg J.B., The effect of photospheric heavy elements on the hot DA white dwarf temperature scale, Mon. Not. R. Astron. Soc., 299, 520-534, 1998.

Bhatia, A.K. \& Landi, E., Atomic data and spectral line intensities for Ar XV, At. Data Nucl. Data Tables, 94, 223-256, 2008.

Dimitrijević, M.S., Stark broadening in astrophysics (Applications of Belgrade school results and collaboration with former soviet republics), Astronomical and Astrophysical Transactions, 22, 389412, 2003.

Dimitrijević, M.S. \& Sahal-Bréchot, S., Stark broadening of neutral helium lines, J. Quant. Spectrosc. Radiat. Transfer, 31, 301-313, 1984.

Dimitrijević, M.S. \& Sahal-Bréchot, S., Stark broadening of Mg I spectral lines, Physica Scripta, 52, 41-51, 1995.

Dimitrijević, M.S. et al., On the Stark broadening of Ar XV spectral lines, Proceedings of the VII Bulgarian-Serbian Astronomical Conference (VII BSAC) 1-4 june 2010, Chepelare, Bulgaria. Publ. Astron. Soc. "Rudjer Bošković", vol. 11, pp. 243-246, 2012.

Edlen, B., Comparison of Theoretical and Experimental Level Values of the $\mathrm{n}=2$ Complex in Ions Isoelectronic with $\mathrm{Li}, \mathrm{Be}, \mathrm{O}$ and $\mathrm{F}$, Physica Scripta, 28, 51-67, 1983.

Edlen, B., A Note on the $2 \mathrm{p}^{2}$ Configuration in the Beryllium Isoelectronic Sequenc, Physica Scripta, 32, 86-88, 1985.

Eissner, W., The UCL distorted wave code, Comput. Phys. Commun., 114, 295-341, 1998.

Eissner, W., Jones, M. \& Nussbaumer, H., Techniques for the calculation of atomic structures and radiative data including relativistic corrections, Comput. Phys. Commun., 8, 270-306, 1974.

Elabidi, H., Ben Nessib, N., Cornille, M., Dubau, J. \& Sahal-Bréchot, S., Quantum-mechanical Calculations of Ne VII Spectral Line Widths, Spectral Line Shapes in Astrophysics (Sremski Karlovci, Serbia), edited by L. Č. Popović \& M. S. Dimitrijević, AIP Conf. Proc., No. 938, pp. 272-275, 2007.

Elabidi, H., Ben Nessib, N., Cornille, M., Dubau, J. \& Sahal-Bréchot, S., Electron impact broadening of spectral lines in Be-like ions: quantum calculations, J. Phys. B: At. Mol. Opt. Phys., 41, no 025702, 2008a.

Elabidi, H., Ben Nessib, N., Sahal-Bréchot, S., Quantum mechanical calculations of the electron-impact broadening of spectral lines for intermediate coupling, J. Phys. B: At. Mol. Opt. Phys., 37, 63-71, 2004.

Elabidi, H., Ben Nessib, N. \& Sahal-Bréchot, S., Quantum calculations of Stark broadening of Li-like ions; T and Zscaling, Spectral Line Shapes (Valladolid, Spain), edited by M. A. González \& M. A. Gigosos, AIP Conf. Proc. No. 1058, pp. 146-148, 2008 b.

Elabidi, H. \& Sahal-Bréchot, S., Checking the dependence on the upper level ionization potential of electron impact widths together with corresponding quantum calculations, Eur. Phys. J. D, 61, 285-290, 2011.

Elabidi, H., Sahal-Bréchot, S. \& Ben Nessib, N., Quantum Stark broadening of 3s3p spectral lines in Li-like ions; Z-scaling and 
comparison with semi-classical perturbation theory, Eur. Phys. J. D, 54, 51-64, 2009.

Elabidi, H., Sahal-Brechot, S. \& Ben Nessib, N., Fine structure collision strengths for S VII lines,Phys. Scr., 85, 065302, 2012.

Elabidi, H., Sahal-Bréchot, S., Dimitrijević, M.S. \& Ben Nessib, N., Quantum Stark broadening data for the C IV, N V, O VI, F VII and Ne VIII resonance doublets, Mon. Not. R. Astron. Soc., 417, 2624-2630, 2011.

Fleurier, C., Sahal-Bréchot, S. \& Chapelle, J., Stark profiles of some ion lines of alkaline earth elements, J. Quant. Spectrosc. Radiat. Transfer, 17, 595-604, 1977.

Khardi, S. et al., Beam-foil spectroscopy in the extreme UV of highly ionized silicon Si XI and the isoelectronic ions AI X, S XIII and Ar XV, Physica Scripta, 49, 571-577, 1994.

Kramida, A., Ralchenko, Yu.V., Reader, J., and NIST ASD Team (2012). NIST Atomic Spectra Database (ver. 5.0), [Online]. Available: http://physics.nist.gov/asd.

Lepson, J.K., Beiersdorfer, P., Behar, E. \& Kahn, S.M., EmissionLine Spectra of Ar IX-Ar XVI in the Soft X-Ray Region 20-50, Astrophys. J., 590, 604-607, 2003.

Ralchenko, Yu.V., Griem, H.R. \& Bray, I., Electron-impact broadening of the 3s-3p lines in low-Z Li-like ions, J. Quant. Spectrosc. Radiat. Tranfer, 81, 371-384, 2003.

Ralchenko, Yu.V., Griem, H.R., Bray, I. \& Fursa, D.V., Electron collisional broadening of 2s3s-2s3p lines in Be-like ions, J. Quant. Spectrosc. Radiat. Tranfer, 71, 595-607, 2001.

Sahal-Bréchot, S., Impact Theory of the Broadening and Shift of Spectral Lines due to Electrons and Ions in a Plasma, A\&A, 1, 91-123, 1969a.

Sahal-Bréchot, S., Impact Theory of the Broadening and Shift of Spectral Lines due to Electrons and Ions in a Plasma (Continued), A\&A, 2, 322-354, 1969b.

Sahal-Bréchot, S., Stark Broadening of Isolated Lines in the Impact Approximation, A\&A, 35, 319-321, 1974.

Saraph, H.E., Fine structure cross sections from reactance matrices a more versatile development of the program JAJOM, Comput. Phys. Commun., 15, 247-258, 1978.

Werner, K., Rauch, T. \& Kruk, J.W., Discovery of photospheric argon in very hot central stars of planetary nebulae and white dwarfs, A\&A, 466, 317-322, 2007. 
Table 1: Our energies in $\mathrm{cm}^{-1}$ (Present) for the lowest 20 levels of Ar XV compared to other results. Exp: experimental energies in Edlen (1983, 1985); Khardi et al. (1994); Lepson et al. (2003) and taken from Bhatia and Landi (2008). NIST: energies from the database NIST (Kramida et al., 2012), Bhatia08: calculated energies with a 27-configuration model (Bhatia and Landi, 2008). $i$ labels the 20 levels. The NIST energies of the two levels 18 and 19 (designed by asterisks) are inverted compared to all the other results.

\begin{tabular}{|c|c|c|c|c|c|c|}
\hline \multicolumn{7}{|c|}{ Level designation } \\
\hline$i$ & Conf. & Level & Present & Exp. & NIST & Bhatia08 \\
\hline 1 & $1 s^{2} 2 s^{2}$ & ${ }^{1} \mathrm{~S}_{0}$ & 0 & 0 & & 0 \\
\hline 2 & $1 s^{2} 2 s 2 p$ & ${ }^{3} \mathrm{P}_{0}^{\mathrm{o}}$ & 228727 & 228674 & 228684 & 229202 \\
\hline 3 & $1 s^{2} 2 s 2 p$ & ${ }^{3} \mathrm{P}_{1}^{\mathrm{o}}$ & 236470 & 235863 & 235860.2 & 236662 \\
\hline 4 & $1 s^{2} 2 s 2 p$ & ${ }^{3} \mathrm{P}_{2}^{\mathrm{o}}$ & 253842 & 252683 & 252679.6 & 254115 \\
\hline 5 & $1 s^{2} 2 s 2 p$ & ${ }^{1} \mathrm{P}_{1}^{\mathrm{o}}$ & 459530 & 452212 & 452182 & 459911 \\
\hline 6 & $1 \mathrm{~s}^{2} 2 \mathrm{p}^{2}$ & ${ }^{3} \mathrm{P}_{0}$ & 608399 & 604961 & 604917 & 609224 \\
\hline 7 & $1 s^{2} 2 p^{2}$ & ${ }^{3} \mathrm{P}_{1}$ & 618807 & 615128 & 615140 & 619718 \\
\hline 8 & $1 \mathrm{~s}^{2} 2 \mathrm{p}^{2}$ & ${ }^{3} \mathrm{P}_{2}$ & 633295 & 628292 & 628308 & 633409 \\
\hline 9 & $1 s^{2} 2 p^{2}$ & ${ }^{1} \mathrm{D}_{2}$ & 698851 & 689621 & & 699392 \\
\hline 10 & $1 \mathrm{~s}^{2} 2 \mathrm{p}^{2}$ & ${ }^{1} \mathrm{~S}_{0}$ & 854805 & 840612 & 840620 & 855441 \\
\hline 11 & $1 s^{2} 2 s 3 s$ & ${ }^{3} \mathrm{~S}_{1}$ & 3938369 & 3935000 & & 3938375 \\
\hline 12 & $1 \mathrm{~s}^{2} 2 \mathrm{~s} 3 \mathrm{~s}$ & ${ }^{1} \mathrm{~S}_{0}$ & 3983232 & 3980000 & 3980760 & 3981941 \\
\hline 13 & $1 s^{2} 2 s 3 p$ & ${ }^{3} \mathrm{P}_{1}^{\mathrm{o}}$ & 4044723 & 4042037 & & 4044306 \\
\hline 14 & $1 s^{2} 2 s 3 p$ & ${ }^{3} \mathrm{P}_{0}^{\mathrm{o}}$ & 4046486 & 0 & & 4045888 \\
\hline 15 & $1 s^{2} 2 s 3 p$ & ${ }^{1} \mathrm{P}_{1}^{\mathrm{o}}$ & 4051014 & 4042600 & 4042040 & 4050223 \\
\hline 16 & $1 s^{2} 2 s 3 p$ & ${ }^{3} \mathrm{P}_{2}^{\mathrm{o}}$ & 4053511 & 4050500 & & 4052584 \\
\hline 17 & $1 \mathrm{~s}^{2} 2 \mathrm{~s} 3 \mathrm{~d}$ & ${ }^{3} \mathrm{D}_{1}$ & 4111931 & 0 & 4106160 & 4110053 \\
\hline 18 & $1 \mathrm{~s}^{2} 2 \mathrm{~s} 3 \mathrm{~d}$ & ${ }^{3} \mathrm{D}_{2}$ & 4112940 & 0 & $4113330 *$ & 4111049 \\
\hline 19 & $1 \mathrm{~s}^{2} 2 \mathrm{~s} 3 \mathrm{~d}$ & ${ }^{3} \mathrm{D}_{3}$ & 4114464 & 4110000 & $4109660 *$ & 4112559 \\
\hline 20 & $1 \mathrm{~s}^{2} 2 \mathrm{~s} 3 \mathrm{~d}$ & ${ }^{1} \mathrm{D}_{2}$ & 4158547 & 4150000 & 4149860 & 4155932 \\
\hline
\end{tabular}


Table 2: Weighted oscillator strengths $g * f$ and collision strengths $\Omega$ for transitions from the lowest five levels to the lowest 10 ones. Present: the present results, Bhatia08: calculated values from Bhatia and Landi (2008) with the 27-configuration model.

\begin{tabular}{|c|c|c|c|c|c|c|}
\hline \multirow{2}{*}{$\begin{array}{c}\text { Transition } \\
i-j\end{array}$} & \multicolumn{2}{|c|}{ Oscillator strengths $(g * f)$} & \multicolumn{2}{|c|}{$\Omega(10 \mathrm{Ry})$} & \multicolumn{2}{|c|}{$\Omega(180 \mathrm{Ry})$} \\
\hline & Present & Bhatia08 & Present & Bhatia08 & Present & Bhatia08 \\
\hline $1-2$ & & & $2.898 \mathrm{E}-03$ & $3.064 \mathrm{E}-03$ & $3.530 \mathrm{E}-04$ & $3.596 \mathrm{E}-04$ \\
\hline $1-3$ & $2.290 \mathrm{E}-04$ & $1.203 \mathrm{E}-04$ & $8.693 \mathrm{E}-03$ & $1.146 \mathrm{E}-02$ & $4.256 \mathrm{E}-03$ & $4.305 \mathrm{E}-03$ \\
\hline $1-4$ & & & $1.449 \mathrm{E}-02$ & $1.507 \mathrm{E}-02$ & $1.735 \mathrm{E}-03$ & $1.765 \mathrm{E}-03$ \\
\hline $1-5$ & $2.090 \mathrm{E}-01$ & $2.093 \mathrm{E}-01$ & $6.753 \mathrm{E}-01$ & $6.682 \mathrm{E}-01$ & $7.623 \mathrm{E}-01$ & $1.197 \mathrm{E}+00$ \\
\hline $1-6$ & & & $1.510 \mathrm{E}-04$ & $1.595 \mathrm{E}-04$ & $2.600 \mathrm{E}-05$ & $2.766 \mathrm{E}-05$ \\
\hline $1-7$ & & & $4.520 \mathrm{E}-04$ & $3.878 \mathrm{E}-04$ & $2.100 \mathrm{E}-05$ & $2.207 \mathrm{E}-05$ \\
\hline $1-8$ & & & $7.540 \mathrm{E}-04$ & $8.283 \mathrm{E}-04$ & $2.150 \mathrm{E}-04$ & $2.274 \mathrm{E}-04$ \\
\hline $1-9$ & & & $3.374 \mathrm{E}-03$ & $4.802 \mathrm{E}-03$ & $4.585 \mathrm{E}-03$ & $4.848 \mathrm{E}-03$ \\
\hline $1-10$ & & & $1.317 \mathrm{E}-03$ & $1.310 \mathrm{E}-03$ & $1.021 \mathrm{E}-03$ & $1.192 \mathrm{E}-03$ \\
\hline $2-3$ & & & $3.131 \mathrm{E}-02$ & $3.289 \mathrm{E}-02$ & $3.066 \mathrm{E}-03$ & $3.085 \mathrm{E}-03$ \\
\hline $2-4$ & & & $2.220 \mathrm{E}-02$ & $2.172 \mathrm{E}-02$ & $1.776 \mathrm{E}-02$ & $1.764 \mathrm{E}-02$ \\
\hline $2-5$ & & & $7.731 \mathrm{E}-03$ & $7.963 \mathrm{E}-03$ & $5.970 \mathrm{E}-04$ & $6.016 \mathrm{E}-04$ \\
\hline $2-6$ & & & $1.771 \mathrm{E}-03$ & $1.988 \mathrm{E}-03$ & $2.140 \mathrm{E}-04$ & $2.170 \mathrm{E}-04$ \\
\hline $2-7$ & $8.158 \mathrm{E}-02$ & $8.167 \mathrm{E}-02$ & $3.286 \mathrm{E}-01$ & $3.192 \mathrm{E}-01$ & $3.536 \mathrm{E}-01$ & $5.789 \mathrm{E}-01$ \\
\hline $2-8$ & & & $2.214 \mathrm{E}-03$ & $3.664 \mathrm{E}-03$ & $3.900 \mathrm{E}-04$ & $4.094 \mathrm{E}-04$ \\
\hline $2-9$ & & & $4.695 \mathrm{E}-03$ & $3.662 \mathrm{E}-03$ & $3.850 \mathrm{E}-04$ & $3.929 \mathrm{E}-04$ \\
\hline $2-10$ & & & $5.490 \mathrm{E}-04$ & $4.368 \mathrm{E}-04$ & $3.600 \mathrm{E}-05$ & $3.616 \mathrm{E}-05$ \\
\hline $3-4$ & & & $8.910 \mathrm{E}-02$ & $8.868 \mathrm{E}-02$ & $4.333 \mathrm{E}-02$ & $4.340 \mathrm{E}-02$ \\
\hline $3-5$ & & & $2.319 \mathrm{E}-02$ & $2.417 \mathrm{E}-02$ & $2.074 \mathrm{E}-03$ & $2.091 \mathrm{E}-03$ \\
\hline $3-6$ & $7.775 \mathrm{E}-02$ & $7.792 \mathrm{E}-02$ & $3.286 \mathrm{E}-01$ & $3.242 \mathrm{E}-01$ & $5.866 \mathrm{E}-01$ & $5.880 \mathrm{E}-01$ \\
\hline $3-7$ & $5.972 \mathrm{E}-02$ & $5.984 \mathrm{E}-02$ & $2.534 \mathrm{E}-01$ & $2.466 \mathrm{E}-01$ & $2.655 \mathrm{E}-01$ & $4.361 \mathrm{E}-01$ \\
\hline $3-8$ & $1.041 \mathrm{E}-01$ & $1.041 \mathrm{E}-01$ & $4.158 \mathrm{E}-01$ & $4.025 \mathrm{E}-01$ & $4.409 \mathrm{E}-01$ & $7.225 \mathrm{E}-01$ \\
\hline $3-9$ & $7.344 \mathrm{E}-04$ & $7.387 \mathrm{E}-04$ & $1.409 \mathrm{E}-02$ & $1.574 \mathrm{E}-02$ & $4.123 \mathrm{E}-03$ & $5.680 \mathrm{E}-03$ \\
\hline $3-10$ & $6.809 \mathrm{E}-05$ & $6.917 \mathrm{E}-05$ & $1.646 \mathrm{E}-03$ & $1.655 \mathrm{E}-03$ & $2.950 \mathrm{E}-04$ & $3.828 \mathrm{E}-04$ \\
\hline $4-5$ & & & $3.866 \mathrm{E}-02$ & $4.095 \mathrm{E}-02$ & $3.162 \mathrm{E}-03$ & $3.181 \mathrm{E}-03$ \\
\hline $4-6$ & & & $2.214 \mathrm{E}-03$ & $2.015 \mathrm{E}-03$ & $2.150 \mathrm{E}-04$ & $2.326 \mathrm{E}-04$ \\
\hline $4-7$ & $9.494 \mathrm{E}-02$ & $8.517 \mathrm{E}-02$ & $4.158 \mathrm{E}-01$ & $4.095 \mathrm{E}-01$ & $4.432 \mathrm{E}-01$ & $7.369 \mathrm{E}-01$ \\
\hline $4-8$ & $2.871 \mathrm{E}-01$ & $2.871 \mathrm{E}-02$ & $1.245 \mathrm{E}+00$ & $1.164 \mathrm{E}+00$ & $1.281 \mathrm{E}+00$ & $2.116 \mathrm{E}+00$ \\
\hline $4-9$ & $1.170 \mathrm{E}-02$ & $1.168 \mathrm{E}-02$ & $2.348 \mathrm{E}-03$ & $6.612 \mathrm{E}-02$ & $4.662 \mathrm{E}-02$ & $7.232 \mathrm{E}-02$ \\
\hline $4-10$ & & & $2.743 \mathrm{E}-03$ & $3.441 \mathrm{E}-03$ & $3.060 \mathrm{E}-04$ & $3.103 \mathrm{E}-04$ \\
\hline $5-6$ & $2.182 \mathrm{E}-04$ & $2.214 \mathrm{E}-04$ & $2.790 \mathrm{E}-03$ & $5.106 \mathrm{E}-03$ & $2.934 \mathrm{E}-03$ & $5.598 \mathrm{E}-03$ \\
\hline $5-7$ & $6.686 \mathrm{E}-05$ & $6.663 \mathrm{E}-05$ & $8.371 \mathrm{E}-03$ & $9.420 \mathrm{E}-03$ & $1.693 \mathrm{E}-03$ & $2.430 \mathrm{E}-03$ \\
\hline $5-8$ & $4.230 \mathrm{E}-03$ & $4.198 \mathrm{E}-03$ & $1.395 \mathrm{E}-02$ & $6.147 \mathrm{E}-02$ & $4.492 \mathrm{E}-02$ & $8.579 \mathrm{E}-02$ \\
\hline $5-9$ & $2.363 \mathrm{E}-01$ & $2.356 \mathrm{E}-01$ & $1.776 \mathrm{E}+00$ & $1.660 \mathrm{E}+00$ & $1.697 \mathrm{E}+00$ & $3.127 \mathrm{E}+00$ \\
\hline $5-10$ & $1.485 \mathrm{E}-01$ & $1.490 \mathrm{E}-01$ & $5.802 \mathrm{E}-01$ & $5.506 \mathrm{E}-01$ & $6.374 \mathrm{E}-01$ & $1.036 \mathrm{E}+00$ \\
\hline
\end{tabular}


Table 3: Present quantum (Q) and semiclassical (SCP) line widths of some Ar XV transitions involving the ground level.

\begin{tabular}{ccccc}
\hline Transition & $\mathrm{T}\left(10^{5} \mathrm{~K}\right)$ & $Q\left(10^{-3} \AA\right)$ & $S C P\left(10^{-3} \AA\right)$ & $\frac{S C P}{Q}$ \\
\hline $1 s^{2} 2 s^{21} \mathrm{~S}_{0}-2 s 2 p{ }^{1} \mathrm{P}_{1}^{0}$ & 5 & 8.550 & 14.3 & 1.67 \\
$\lambda=221.15 \AA$ & 7.5 & 7.660 & 11.7 & 1.53 \\
& 10 & 7.040 & 10.2 & 1.45 \\
$1 s^{2} 2 s^{2}{ }^{1} \mathrm{~S}_{0}-2 s 3 p{ }^{1} \mathrm{P}_{1}^{0}$ & 5 & 5.620 & 7.46 & 1.33 \\
$\lambda=24.7 \AA$ & 7.5 & 0.242 & 0.455 & 1.88 \\
& 10 & 0.209 & 0.373 & 1.78 \\
$1 s^{2} 2 s^{2}{ }^{1} \mathrm{~S}_{0}-2 s 4 p{ }^{1} \mathrm{P}_{1}^{0}$ & 20 & 0.141 & 0.325 & 1.73 \\
$\lambda=18.8 \AA$ & 7.5 & 0.498 & 0.235 & 1.67 \\
& 10 & 0.398 & 0.758 & 1.52 \\
$1 s^{2} 2 s^{2}{ }^{1} \mathrm{~S}_{0}-2 s 5 p{ }^{1} \mathrm{P}_{1}^{0}$ & 5 & 0.224 & 0.634 & 1.59 \\
$\lambda=16.95 \AA$ & 7.5 & 0.884 & 0.422 & 1.66 \\
& 10 & 0.583 & 1.37 & 1.55 \\
& 20 & 0.370 & 1.04 & 1.69 \\
& 5 & & 0.805 & 1.79 \\
\hline
\end{tabular}

Table 4: Present quantum (Q) and semiclassical (SCP) line widths of two Ar XV transitions between excited levels.

\begin{tabular}{ccccc}
\multicolumn{2}{c}{ of two Ar XV transitions between excited levels. } \\
\hline Transition & $\mathrm{T}\left(10^{5} \mathrm{~K}\right)$ & $Q\left(10^{-3} \AA\right)$ & $S C P\left(10^{-3} \AA\right)$ & $\frac{S C P}{Q}$ \\
\hline $1 s^{2} 2 s 2 p{ }^{3} \mathrm{P}_{0}^{0}-2 s 3 s^{3} \mathrm{~S}_{1}$ & 5 & 0.331 & 0.242 & 0.73 \\
$\lambda=27.0 \AA$ & 7.5 & 0.273 & 0.202 & 0.74 \\
& 10 & 0.236 & 0.177 & 0.75 \\
$1 s^{2} 2 s 2 p^{1} \mathrm{P}_{1}^{0}-2 s 3 s{ }^{1} \mathrm{~S}_{0}$ & 5 & 0.164 & 0.133 & 0.81 \\
$\lambda=28.4 \AA$ & 7.5 & 0.363 & 0.280 & 0.77 \\
& 10 & 0.309 & 0.232 & 0.75 \\
& 20 & 0.197 & 0.204 & 0.75 \\
& & & 0.152 & 0.77 \\
\hline
\end{tabular}

Table 5: Strong collisions (strong) and quadrupolar potential (quad) contributions to line widths.

\begin{tabular}{|c|c|c|c|}
\hline & strong/total $(\%)$ & quad $/ \operatorname{total}(\%)$ & $\frac{|S C P-Q|}{Q}(\%)$ \\
\hline $2 s^{21} \mathrm{~S}_{0}-2 s 2 p{ }^{1} \mathrm{P}_{1}^{0}$ & 42 & 65 & 67 \\
\hline $2 s^{2}{ }^{1} \mathrm{~S}_{0}-2 s 3 p^{1} \mathrm{P}_{1}^{0}$ & 56 & 88 & 88 \\
\hline $2 s^{2}{ }^{1} \mathrm{~S}_{0}-2 s 4 p^{1} \mathrm{P}_{1}^{0}$ & 45 & 71 & 52 \\
\hline $2 s^{2}{ }^{1} \mathrm{~S}_{0}-2 s 5 p^{1} \mathrm{P}_{1}^{0}$ & 38 & 60 & 55 \\
\hline $2 s 2 p{ }^{3} \mathrm{P}_{0}^{0}-2 s 3 s{ }^{3} \mathrm{~S}_{1}$ & 35 & 56 & 27 \\
\hline $2 s 2 p^{1} \mathrm{P}_{1}^{0}-2 s 3 s^{1} \mathrm{~S}_{0}$ & 36 & 56 & 23 \\
\hline
\end{tabular}

